\title{
Haptic Communication to Support Biopsy Procedures Learning in Virtual Environments
}

Amine Chellali : Robotics research group, IRCCyN-Ecole des Mines de Nantes and Department of Surgery, Cambridge Health Alliance

Cedric Dumas: Robotics research group, IRCCyN-Ecole des Mines de Nantes and Biomedical Imaging Group, CSIRO

Isabelle Milleville-Pennel: PsyCoTech research group, IRCCyN-CNRS

* Corresponding author:

Email: amine.chellali@gmail.com

Mailing Address:

Cambridge Health Alliance

Department of Surgery Administration

1493, Cambridge Street

Cambridge, MA 02139 - USA

Phone (office): 617-665-3557

Fax (office): 617-627-3058 


\section{ABSTRACT}

In Interventional Radiology, physicians require high haptic sensitivity and fine motor skills development because of the limited real-time visual feedback of the surgical site. The transfer of this type of surgical skills to novices is a challenging issue. This paper presents a study on the design of a biopsy procedure learning system. Our methodology, based on a task-centered design approach, aims to bring out new design rules for virtual learning environments. A new collaborative haptic training paradigm is introduced to support human haptic interaction in a virtual environment. The interaction paradigm supports haptic communication between two distant users to teach a surgical skill. In order to evaluate this paradigm, a user experiment was conducted. Sixty volunteer medical students participated in the study to assess the influence of the teaching method on their performance in a biopsy procedure task. The results show that to transfer the skills, the combination of haptic communication with verbal and visual communications improves the novices' performance compared to conventional teaching methods. Furthermore, the results show that, depending on the teaching method, participants developed different needle insertion profiles. We conclude that our interaction paradigm facilitates expert-novice haptic communication and improves skills transfer; and new skills acquisition depends on the availability of different communication channels between experts and novices. Our findings indicate that the traditional fellowship methods in surgery should evolve to an off-patient collaborative environment that will continue to support visual and verbal communication, but also haptic communication, in order to achieve a better and more complete skills training.

\section{INTRODUCTION}

Teaching a new skill consists of transferring the expert's knowledge to a set of learners. The choice of the appropriate communication channel (voice, vision, or haptics) to transfer the knowledge depends on the skill to be taught and on the available communication mediums. For example, a practitioner acquires fine motor skills through several years of practice. In order to teach his skills to others, he must be able to describe accurately his abilities. This includes information about the speed of movements, the accuracy, and the sharpness of touch. Teaching correctly these skills requires the use of the haptic communication channel as well as the other communication channels. While verbal and visual communication channels are widely studied, human haptic communication for skills transfer needs to be more investigated. In this context, Virtual Reality (VR) technologies offer a promising teaching tool. Indeed, virtual environments that include haptic devices provide a safe and versatile practice medium to teach motor abilities.

The aim of this research is to investigate the role of haptic collaboration for teaching fine motor skills in virtual environments. The main motivation of this work is the need for systems that help practitioners (surgeons and radiologists) transfer their knowledge. Our methodology is based on a task-centered design approach to develop teaching systems. For that purpose, a new collaborative haptic training paradigm is used (Chellali, Milleville, \& Dumas, 2010). It allows real-time visual, verbal, and haptic communication between an expert and a novice. It allows them to better interact and to exchange information in the early learning stages. To demonstrate the concept, we conducted a user experiment in which 
participants were taught how to perform a biopsy procedure. Before introducing our experimental approach, an overview of the existing works in this area is presented.

\section{PROBLEMATIC}

\subsection{Communication channels for teaching new skills}

Learning may be considered a collaborative process in which experts and novices work together toward a common goal: knowledge transfer. To achieve this goal, both operators must build a common ground: a common and consistent representation of the shared situation (Clark \& Brennan, 1991). In fact, during the learning process, the novice constructs his own individual but incomplete representation of the situation. The expert then tries to complete and enrich the novice's representation by teaching the new skill. In a motor skills teaching situation, it is essential to allow the expert to accurately communicate his knowledge. For that, he can use three different communication channels (Gillespie, O'Modhrain, Tang, Zaretsky, \& Pham, 1998):

\subsubsection{Verbal communication}

Verbal communication is the immediate and first mean of knowledge transfer, and allows the correct rules for the skill execution to be easily shared (declarative knowledge). However, it cannot be used alone to efficiently transfer the motor skills properties. Indeed, motor skills involve invisible elements such as tactile and haptic sensations (that belong to the procedural knowledge) which are difficult to describe verbally (Rasmussen, 1983).

\subsubsection{Visual communication (demonstration)}

The teacher demonstrates the skill by showing a visual model of the movement to perform. This allows the teacher to share more easily the skills since he communicates his knowledge while acting. Learners observe the visual model and then try to reproduce the movement. However, this model gives an incomplete representation of the skill. In fact, only the posture and the movements' dynamics are shown. The cause-and-effect of the movement principles cannot fully be demonstrated. This may encourage imitation rather than true learning. In addition, the learner must retrieve haptic information from the visual coordinate system. This is likely to be difficult for complex tasks such as surgical skills, particularly in the early learning stages.

\subsubsection{Haptic communication (hand-guidance)}

Hand-guidance can be observed when an expert takes the novice's hand to show the correct movement. In this case, the direct contact between their hands permits communication of the knowledge. This is referred to as haptic communication that allows for the exchange of information about the forces and the movement dynamics. It leads to a richer model of interaction between the expert and the novice since it facilitates the sharing of more information while acting. However, it suffers from some restrictions; an expert can only work with one novice at a time. Moreover, the contact between the expert's hand and the novice's hand modifies their perception of the movement and environment feedback. This may alter the main task performance. Finally, many previous researches (see (Lee \& Choi, 2010) for a review) have shown that haptic guidance is ineffective, or sometimes even detrimental, for learning basic motor skills. Nevertheless, the surgical skills need to be considered differently. 
Indeed, senior surgeons rank the cognitive (such as decision making) as the most important skill of a surgeon, above all technical skills (Baldwin, Paisley, \& Brown, 1999; Cuschier, 2011). Therefore, we hypothesize that for complex tasks (such as surgical skills) the guidance is necessary: when the execution of the motor component of the task is hardly dependant on the cognitive component, the interaction with a teacher in the early learning stages becomes essential to help the leaner complete his own mental model of the task (Payne, 2009).

This review shows that haptic communication is required to transfer motor abilities. However, the other channels are necessary to complete the novice's mental representation of the skill. Hence, to build efficient learning systems that support both technical and cognitive skills transfer, it is important to enrich the interaction between experts and novices by the combination of several communication channels. The Virtual environments that support haptics are regarded as rich interaction mediums that serve such a purpose.

\subsection{Virtual Reality to support learning}

Before using virtual environments as a teaching medium, it is necessary to investigate their influence on human haptic interactions. VR technology has a history of use for many training applications such as military training, surgical training, and training in spatial awareness (Burdea \& Coiffet, 2003). Several commercial systems such as the MIST-VR, developed to train complex surgical skills, have been shown to significantly improve the human motor performance (Gallagher, McClure, McGruigan, Crothers, \& Browning, 1999). Virtual environments have also been shown to be excellent promise as a tool for rehabilitative movement retraining (see (Merians, Poizner, Boian, Burdea, \& Adamovich, 2006) for a more extensive review).

However, despite the use of VR technologies for skills learning, little research addresses the usability of haptic communication in virtual environments. We hypothesize that this communication channel is a key component to efficiently teach new surgical skills. Previous studies (Basdogan, Ho, Srinivasan, \& Slater, 2000; Sallnäs, Rassmus-Gröhn, \& Sjöström, 2000) suggest that haptic communication improves users' performance in manual collaborative tasks. However, the nature of information exchanged through the haptic channel and its effects on learning has not yet been investigated.

In this paper we investigate the role of multimodal interaction used to improve communication during skills transfer. Our approach is supported by the common-ground theory: efficient communication improves mutual understanding between the expert and the novice (Clark \& Brennan, 1991). Before addressing this issue, we must understand how haptic communication can influence the transfer of skills in a collaborative training situation.

\subsection{Haptic training: providing haptic information in motor skills learning}

The importance of haptic information for learning new motor skills remains an open issue (Blavier, 2006). Some authors have shown the importance of haptic information for teaching motor skills (Bluteau, Coquillart, Payan, \& Gentaz, 2008; Solis, Avizzano, \& Bergamasco, 2003). On the other hand, other researchers question the necessity of this information. For instance, Willingham (1998) argue that learning can take place when haptic information is not available (in learning by observation for example). Zhou et al. (2008) suggested that 
haptic feedback may be omitted in surgical simulators provided that extensive training is possible.

We present in this section a review of self-learning applications that support haptic interaction to teach motor skills. We will focus in this section on haptic communication systems, since we hypothesize that it will improve the learning of surgical skills. Many existing systems reproduce the hand guidance paradigm (Feygin, Keehner, \& Tendick, 2002) and are based on the record and replay metaphor. The implementation of this paradigm in an automatic teaching system requires recording and modeling an expert's reference gesture. This reference gesture is then "replayed" to the learner through a haptic device. This was used in many applications such as in a calligraphy learning system (Yoshikawa \& Henmi, 2000), a crane-moving learning task (Gillespie, O'Modhrain, Tang, Zaretsky, \& Pham, 1998), and for memorizing a sequence of forces (Morris, Tan, Barbagli, Chang, \& Salisbury, 2007). These studies show that haptic guidance combined with visual guidance allows novices to learn more effectively some motor skills properties compared to visual-guidance-only systems.

However, the use of an automatic system for teaching presents some restrictions. Hand guidance can prevent error-based learning: the novice cannot learn from his own mistakes. Furthermore, a haptic guidance system can transmit information about positions or forces, but not both at once. Finally, it does not support direct interaction with a human expert. This prevents the novice from asking questions and the expert from providing further explanations and limits the development of the novice's personal representation. In this context, a shared control paradigm for training in minimally invasive surgery was presented (Nudehi, Mukherjee, \& Ghodoussi, 2003). The system allows an expert surgeon to interact with a trainee by sharing the control of a robot when performing a surgical procedure. However, no human subject study was reported to support this paradigm. A similar system was presented by Esen et al. (Esen, Sachsenhauser, Yano, \& Buss, 2007) for bone drilling medical training. Different collaborative strategies for teaching a trainee the correct forces to apply were presented. The authors conducted user tests that have shown the efficiency of the training methods to teach reference forces. However, no investigation on teaching the cognitive skills related to the bone drilling task was presented. Gunn et al. (2005) presented another hand guidance scenario for surgical training. The system allowed an expert and a novice to collaborate to perform a cholecystectomy procedure (removal of the gallbladder). However, the focus in this work was on network latency and no evaluation of the skills transfer was presented. Finally, Lathan et al. (2000) developed a VR simulator for spin biopsy procedures. The evaluation of this system has demonstrated the importance of haptic feedback for biopsy procedures especially when the visual feedback is limited. This confirms that haptic information is a key component that needs to be correctly taught in biopsy procedures learning.

In this paper, we present a user-centered design approach for a surgical skill learning system. Instead of an automatic self-training environment, we propose a new haptic collaboration paradigm inspired from the hand guidance metaphor and based on direct interactions with the experts. We hypothesize that compared to record and replay systems, a learning system that permits a direct interaction between an expert and a novice, in the early learning stages, can 
allow them to develop a more efficient common ground and to exchange more accurate information about cognitive and technical skills properties. Furthermore, virtual environments can be designed to support interactions and to improve communication by providing a shared visual workspace.

In the following section, we present our interaction concept, and the user study that aims to evaluate it.

\section{THE COLLABORATIVE HAPTIC TRAINING PARADIGM}

The previous review leads to the following issues:

1 Visual demonstrations give the learner an incomplete mental representation of the skills. Can the expert share a more complete mental representation of his knowledge with novices?

2 Hand-guidance is an interesting alternative for teaching motor skills: direct contact enriches the development of common ground by supporting haptic interactions. However, the metaphor suffers from some restrictions. Can we improve the guidance metaphor to help an expert transmit faithfully his knowledge?

3 The properties of virtual environments can change the way two users interact haptically with each other. How can we support haptic communication in VR environments?

4 The successful transfer of knowledge depends on the communication channel being used. If the appropriate communication channels are not supported by the learning system, this can alter the knowledge transfer. For instance, the record and replay systems are limited to haptic training. How can we support direct multimodal interactions between experts and novices to improve the acquisition of skills?

While addressing the questions above would help to define strong design principles to build an effective learning tool, the limitations of the current technologies constraint the implementation of all the features. Our collaborative haptic training paradigm (Chellali, Milleville, \& Dumas, 2010) implements these principles to some extent. The paradigm is based on the guidance metaphor, extended with the support of three communication channels: verbal, visual and haptic allowing a real-time multimodal interaction between the learner and the teacher in a VR environment.

\subsection{Paradigm principles}

The collaborative haptic training is based on (Erreur ! Source du renvoi introuvable.):

- Two identical tools linked in a master-slave setup.

- Two collaborating users: The actor moves a (master) tool, while the observer passively follows the movements by handling a linked (slave) tool.

- The slave tool is controlled in position by the master.

- Depending on the link between the tools (one-way or bilateral), different interaction scenarios are supported.

- The actor can perform the manual task freely while guiding the observer's hand. 
- Haptic communication is combined with verbal and visual communication; the actor provides the observer the necessary information to learn the technical and cognitive skills through the appropriate channel.

- A single display screen allows the users to share the same viewpoint and workspace of the virtual environment,

- An actor can guide simultaneously several observers.

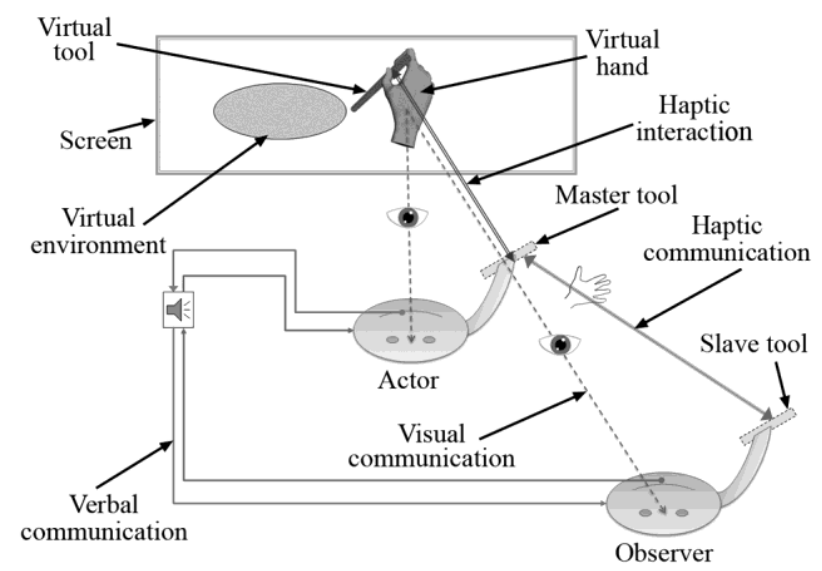

Fig. 1: The collaborative haptic training paradigm: The actor interacts directly with the virtual environment using the master arm (moving the virtual object and feeling the haptic feedback). At the same time, he can guide the observer's hand using three communication channels (verbal, visual, and haptic). The haptic guidance can be one-way or bilateral.

\section{SYSTEM DESIGN}

In order to evaluate the paradigm, a first implementation of the system is presented. This implementation illustrates one possible learning scenario supported by the paradigm. The system consists of a learning environment for a minimally invasive surgical procedure (biopsy). The biopsy procedure was chosen as a test task because of its dependency on declarative knowledge (planning rules) as well as procedural knowledge (haptic and tactile information). Hence, it represents a sufficiently complex task to evaluate the effectiveness of the system. The different steps of the user-centered design are described hereafter.

\subsection{Task analysis}

The first phase of our design methodology included a task analysis which is a standard method in human factors engineering. It was used to determine the key cognitive and technical skills the surgeon needs to successfully complete a biopsy procedure. This critical information was used to guide the design of the user interface. It was used also to define and validate the teaching scenarios and the evaluation measurements in collaboration with the expert surgeons. 


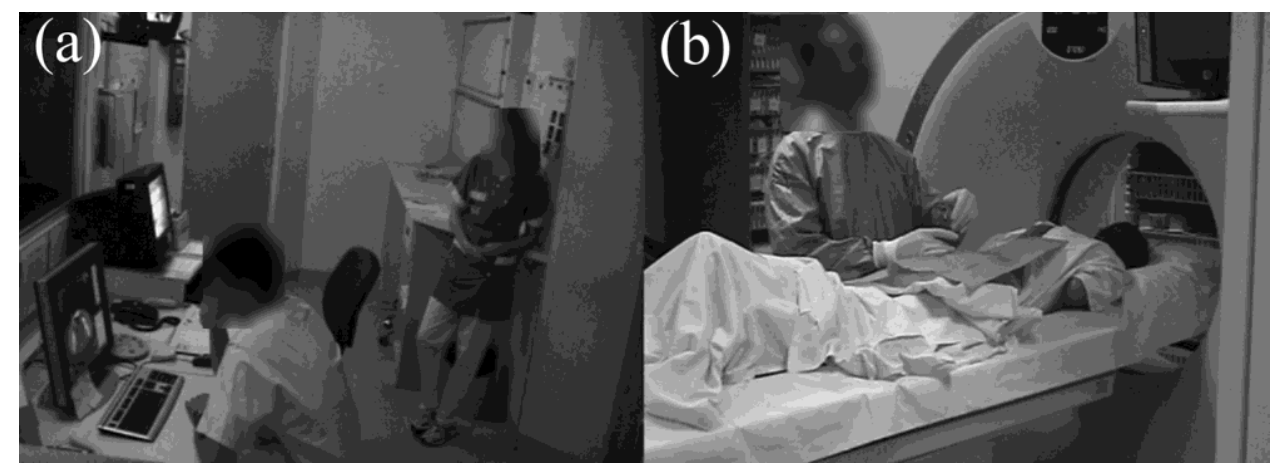

Fig. 2: Biopsy procedure task analysis in the Radiology Department (Teaching hospital of Nantes): (a) analyses of the CT scan images and path planning (b) the needle insertion and path-tracking

The task analysis consisted of observations and video recordings of the radiologists during real biopsies performed (Erreur ! Source du renvoi introuvable.) over a six months period. The radiologists also answered a questionnaire and participated in an individual selfconfrontation (Lim, 2002) video interview session in which they commented their own actions. This analysis phase allowed for a detailed description of the task to be created, including the technical skills (procedural knowledge) and the planning rules (declarative knowledge).

\subsubsection{The biopsy procedure description}

Minimally invasive procedures are performed under image guidance (CT scan, MRI, etc.). Percutaneous needle insertions are commonly used in interventional radiology to perform biopsy or radiofrequency ablations of tumor cells. As a result of the less invasive nature of such procedures, patients generally recover faster. A common task in interventional radiology is tissue biopsy. Biopsies are commonly performed to collect a sample of suspicious cells which is then sent to the laboratory for analysis. To perform a biopsy, the radiologists perform very accurate movements by manipulating a specific tool (a biopsy needle). Prior to the needle insertion, radiologists use CT scan images to locate the target (Erreur ! Source du renvoi introuvable., a) and plan their insertion path. During the operation, the radiologists have no real-time visual feedback of the needle position inside the body (Erreur ! Source du renvoi introuvable., b). Hence, they rely mainly on the haptic feedback and on the spatial memorization of the offline images to insert the needle.

\subsubsection{Task decomposition}

The biopsy procedure can be divided into two main steps:

- Planning phase: the objective is to use the CT scan images to locate the tumor. After that, the radiologist defines an entry point and plans an insertion path to reach the tumor with respect to some physical and safety constraints.

- Manipulation phase: the purpose is to insert the needle inside the patient's body in order to remove a sample of tumor cells. The radiologist follows the defined path until he reaches the tumor. Since he has no real-time visual feedback during needle insertion, he relies mainly on the path memorization and on the haptic sensations when passing across the different tissue layers. 


\subsubsection{Biopsy procedures learning methods}

Minimally invasive procedures imply changes in the way surgeons perform their activities. This introduces a lot of difficulties for skills acquisition and training. Currently, in addition to theoretical courses (verbal communication), the fellows also learn the basic skills for biopsy procedures by observing (visual communication) expert radiologists in real situations. Medical education literature suggests that learning by doing is superior to learning by reading or observation, particularly in relation to hands-on skills. Subsequently, the speed of movements, accuracy, sharpness of the touch, and safety are actually learned while performing on live patients under close supervision by a physician during clinical rotations. However, this learning method introduces some patient safety issues (Stassen, Bonjer, Grimbergen, \& Dankelman, 2005) since using real patients as learning participants is questionable. This restrains the training opportunities for fellows and encourages them to prefer other specialties. Designing new tools to help the surgeons overcome these learning issues becomes necessary. For that reason, studying the biopsy procedure through virtual environments is useful to understand the basics of this kind of skills. It can also highlight the importance of including haptic communication as an additional mean for the transfer of knowledge. This will permit to design more efficient off-patient training tools.

\subsubsection{Critical skills to teach}

After the task analysis phase, the critical skills that need to be taught for biopsy procedures were defined jointly with the expert radiologists. These skills were regrouped in two categories:

\section{Cognitive skills: (declarative knowledge)}

1. Choice of the correct slice view: this subtask consists of choosing the best slice view to visualize the workspace and the target area. The radiologist must know how to choose the optimal position of the CT-scan because it permits to reduce the patient's $\mathrm{X}$-rays expositions during the intervention.

2. Path planning with respect to theoretical rules: This subtask consists of choosing the needle entry point as well as the insertion path. For that purpose, several rules (avoiding lesions, minimizing damage to the organs, position the needle tip in the center of the target...etc.,) were extracted. The radiologist must know this rules that guide him correctly plan his intervention.

\section{Technical skills (procedural knowledge)}

1. Following the defined path: in order to facilitate the intervention, the radiologist needs to follow as much as possible the defined path. For that purpose, the radiologist needs to master the needle manipulations during the insertion phase. In some cases, he must be able to readjust the movements according to the actual situation (complications for example).

2. Detect the passage of the needle from one layer to another: To avoid complications, the radiologist is required to be constantly aware of the needle tip position. As no real-time visual feedback is provided, he relies mainly on spatial memory as well as haptic perception to determine the tip position. Therefore, he must be able detect a change in layer compliance when inserting the needle. 
An efficient training system needs to support the teaching of both skills. While the cognitive skills can be taught verbally, hands-on training is required to correctly master the technical skills. Subsequently, our training system based on multimodal interaction and supporting verbal, visual and haptic communication between the teacher and the leaner is expected to allow both skills to be correctly taught.

\subsection{The virtual environment design}

The Graphical User Interface (GUI) design for the biopsy trainer was driven by the task analysis results.

\subsubsection{Design choices}

High-fidelity VR simulators are expensive due to the use of realistic models. Existing simulators are dedicated to a few specialized procedures and much research is still needed to make them physically and visually more realistic. Soft tissue response is difficult to model and requires many hardware and software resources. Finally, human haptic perception is still not completely understood and, the interpretation of a stimulus in a changing context can lead to different user experiences.

On the other hand, part-task simulators are less expensive and easier to design. They have been shown to be effective if they provide the critical information with respect to performance outcome, aiding in problem-solving and decision-making (Cao, 2001; Hutchins, Adcock, Stevenson, Gunn, \& Krumpholz, 2005).

According to the objective of our system (teaching biopsy skills) and to the arguments set forth above, the VR environment was simplified to be composed mainly of symbolic elements (e.g. patient body, organs). However, visual and haptic deformations of the skin and the organs surrounding membranes were simulated.

\subsubsection{GUI description}

The VR graphics were developed using Virtools 4.0 (from Dassault System). The GUI reproduces the radiologists working environment composed of two main views:

- A planning GUI that includes a slice view of the body (representing CT scan images). The user is allowed to change the slice view using a Shuttle Pro device from Contour Design (Erreur! Source du renvoi introuvable.). The GUI allows for defining the entry point and for planning the insertion path (Erreur ! Source du renvoi introuvable.). 


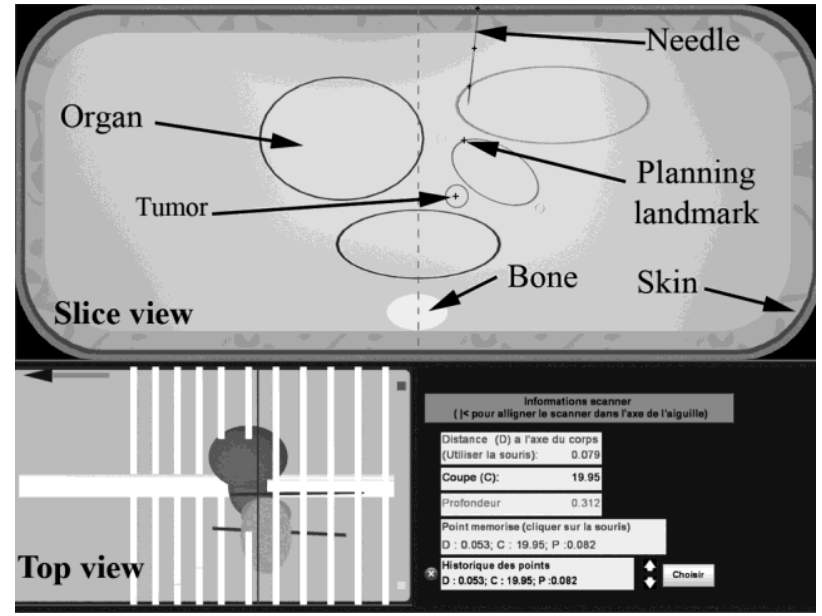

Fig. 3: The planning Graphic User Interface. Users plan the insertion path on the slice view before needle insertion

- A manipulation GUI that allows for insertion of a virtual biopsy needle inside the virtual body using the haptic device. Offline images of slice views were also provided (Erreur ! Source du renvoi introuvable.).

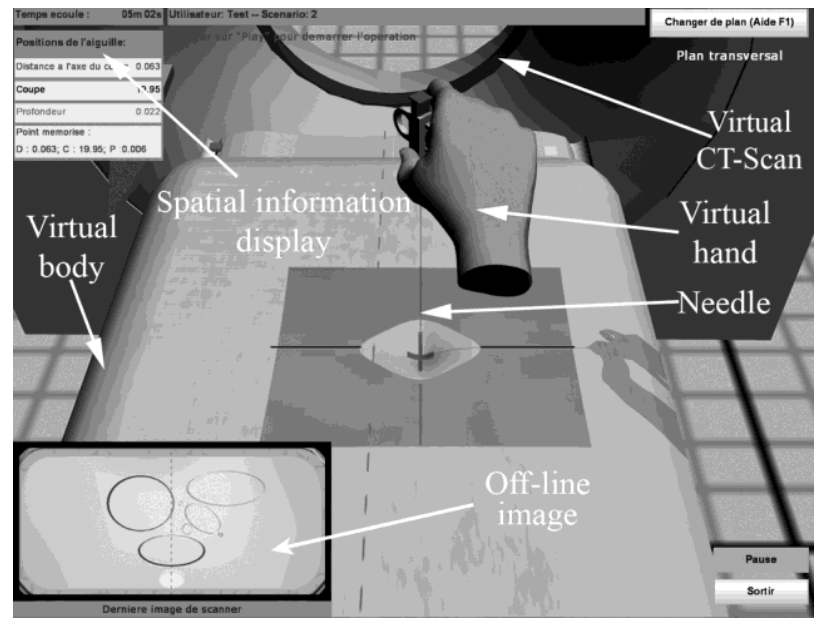

Fig. 4: The manipulation Graphic User Interface. The haptic device is used to insert the virtual needle inside the body

\subsection{Haptic interactions}

To support the user's haptic interactions in the virtual environment, Virtuose $6 \mathrm{D}$ desktop haptic arms from Haption were used. The Virtuose was chosen for this task because of its characteristics:

- It is designed by the French Atomic Energy Commission (CEA) for medical applications.

- It supports feedback of position and force according to $6 \mathrm{DoF}$,

- Its operational workspace matches the movements of the hand $(12 \mathrm{~cm}$ diameter spherical volume),

- The peak forces are $15 \mathrm{~N}$ in translation and $0.5 \mathrm{~N}$ in rotation.

The haptic device was connected to the virtual environment (using the Virtuose API) and was used to manipulate the virtual needle while providing force feedback from the environment. Finally, to make the haptic arm movements match those of a real biopsy needle, its initial 
posture was rotated so that its stylus was in a vertical position (Erreur ! Source du renvoi introuvable.).

\subsubsection{Haptic rendering}

To simplify the haptic rendering, the lateral needle deformations were ignored. To simulate the needle haptic feedback, a multi-layer tissue model was used. The model is inspired from a model of a needle insertion inside a pig liver at a constant speed (Barbé, Bayle, \& de Matheli, 2006). Different model coefficients were assigned to reflect differences in tissue densities (skin, fat, organs, and tumor). Furthermore, the bone was considered as a solid structure that cannot be penetrated.

\subsubsection{Haptic communication}

To support haptic communication, the two haptic arms were linked using a low-latency network connection (Erreur ! Source du renvoi introuvable.). The first arm (master) was used to support haptic interaction with the virtual environment, while the second arm (slave) reproduced the master's movements. To support this haptic link feature, the Virtuose API was used to calculate the master's real-time position and to subsequently move the slave arm so that it reached the same (relative) position.

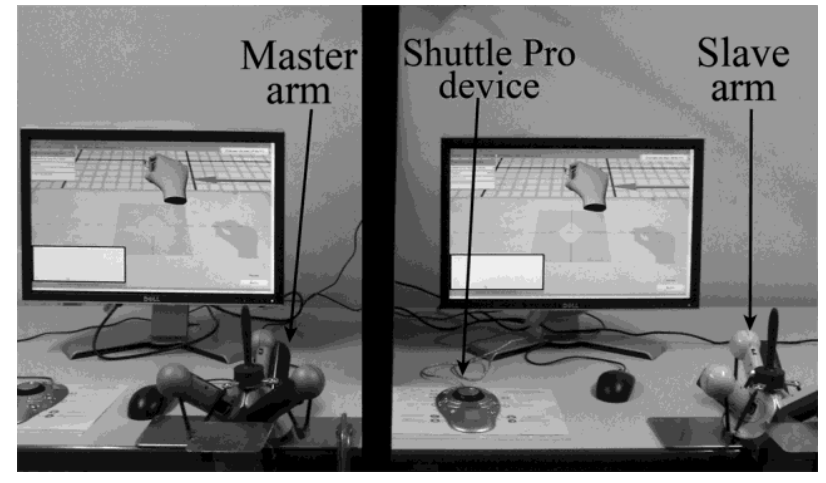

Fig. 5: The haptic communication system with two haptic arms

The master-slave setup was run in a one-way (mono-lateral) control configuration, meaning that only the master was allowed to move the virtual needle: the "observer" had no direct action on the virtual environment.

Finally, several tests were conducted to ensure that the haptic link does not affect the users' interactions with the system. A summary of these tests can be found in (Takac, 2010). The results show that the average time delay between the displacements of the two devices is estimated to be $8 \mathrm{~ms}$. It is stated in the literature (Liukkonen, 2010) that for humans, the effect of time delays for a control task is noticeable around $200 \mathrm{~ms}$. Hence, the delay was ignored for our setup.

\section{USER EVALUATION}

The following user experiment was conducted in order to evaluate the learning system.

\subsection{Learning scenario}

Two distinct users participated in the learning scenario: (i) the actor: an expert in performing biopsy procedures and (ii) the observer: a novice with an interest in learning how to perform the procedure. The task of the expert was to teach the biopsy procedure to the learner by 
guiding him using the haptic communication learning system. This learning method is compared to the conventional learning methods for biopsy (demonstration and theoretical courses) defined during the task analysis phase. The different learning exercises used in the evaluation study were defined in collaboration with the expert radiologists.

\subsection{Hypotheses}

By comparing the haptic communication teaching method to conventional biopsy teaching methods we hypothesize that:

1. H1: Learning the biopsy procedure via haptic communication combined with visual and verbal communication (multimodal learning) can help novices learn the skill faster and perform it more accurately compared to the other methods.

2. H2: The haptic communication method of learning will make the system easier to use compared to the other learning methods.

3. H3: The type of knowledge transferred depends on the communication channel used to teach the skill. Hence, we expect to see different needle insertion strategies depending on the learning method used.

\subsection{Participants}

Sixty (60) novice students (19-29 years old; 30 males, 30 females), from the Medical School of Nantes and from the Veterinary School of Nantes, participated in the study. None of them had prior knowledge of biopsy procedures. In addition, the subjects had neither experience with virtual environments nor with haptic devices. Finally, all of them received a $30 € \mathrm{gift}$ card for their participation.

\subsection{Task and procedure}

The subjects participated in three successive experimental sessions as summarized in Table 1:

Table 1: experimental sessions with time duration

\begin{tabular}{|c|c|c|c|}
\hline & PI & VI & VHI \\
\hline Initial session ( 20min) & \multicolumn{3}{|c|}{60 subjects } \\
\hline Instruction session ( 30min) & 20 subjects & 20 subjects & 20 subjects \\
\hline Practice session ( 90min) & 20 subjects & 20 subjects & 20 subjects \\
\hline
\end{tabular}

\subsubsection{Initial session}

After reading the instructions, the participants were presented with the learning system. The objective of this session was to familiarize them with the manipulation of the haptic device and with the use of the virtual environment so that all of them have the same experience using the haptic device. They were also allowed to perform a simple biopsy scenario. After this initial session, all the participants were observed as feeling comfortable with the experimental setup and with the use of the haptic arm.

\subsubsection{Instruction session}

After the initial session, an instructor (one of the experimenters) taught the participants how to perform correctly a biopsy using four teaching exercises. During these exercises the participants learned how to: 
- Plan the path: The subjects were taught how to choose the needle entry point. After that, they were instructed how to position landmarks in the slice view to determine the insertion path. The path is defined with respect to the planning constraints,

- Manipulate the needle: The subjects were taught the strategies and the constraints that must be considered in order to correctly insert the needle in the virtual body. After that, they were shown how to properly move the needle inside the body to reach the tumor while following the defined path.

Independent variables: Participants were divided into three learning groups and were seated differently depending on these groups:

1. Paper Instructions learning group (PI). The instructor taught both the planning rules and the correct skill to the participants through verbal instructions and with support of static images. The pictures included screenshots of the different needle positions during the procedure and contained necessary information to solve the exercises. This method corresponds to a theoretical teaching method for biopsy (Erreur ! Source du renvoi introuvable.).

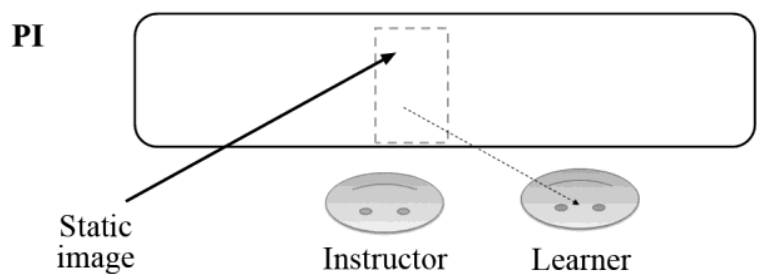

Fig. 6: (PI) Paper Instructions learning condition: the novices were taught the procedure through verbal instructions and with support of static images of the different exercises

2. Visual instructions learning group (VI). In addition of verbal explanations, the instructor demonstrated the strategies and the skill visually. The participants were seated slightly behind the expert. This allowed them to observe directly the expert's hand manipulating the haptic device and to see the visual feedback on the screen. This corresponds to the demonstration teaching method for biopsy (Erreur ! Source du renvoi introuvable.).

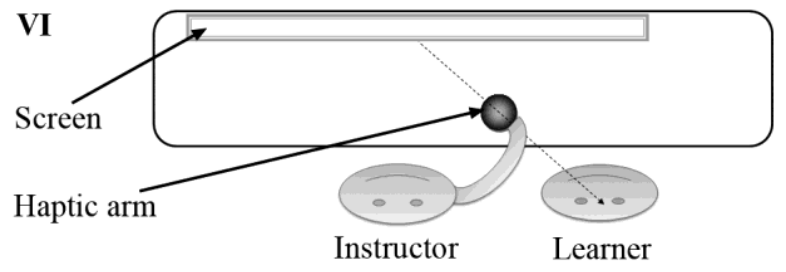

Fig. 7: (VI) Visual Instructions learning condition: the novices were taught the procedure through visual demonstration and verbal instructions

3. Visual-haptic instructions learning group (VHI). In addition to verbal explanations, the instructor demonstrated the strategies and the skill visually. The participants were seated slightly behind the expert in order to see the expert's movements. Furthermore, the instructor used the haptic link to guide the participants' hands while he was manipulating the needle and shared the movements of the needle with them. The haptic arms were connected in a one-way link (a mono-lateral position manipulation). This means that only the instructor was allowed to move the haptic 
arm. Subsequently, the leaner could not intervene in the instructor's performance and could only follow the movements passively (the haptic arm was blocked when the leaner tries to move it). This teaching method corresponds to our initial implementation of the collaborative haptic training paradigm (Erreur ! Source du renvoi introuvable.) that allows the instructor teaching his knowledge by combining three modalities.

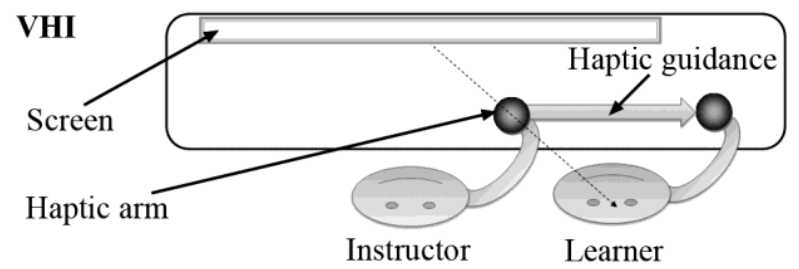

Fig. 8: (VHI) Visual-Haptic Instructions learning condition: the novices were taught the procedure through visual demonstration combined with (one-way) haptic guidance and verbal instructions

\subsubsection{Individual practice session}

In order to assess the learning efficiency, all the subjects performed a practice session. In this session, they were asked to individually perform three new needle insertion scenarios.

\subsection{Evaluation measurements (Dependant variables)}

The measurements were defined with the experts during the task analysis phase. The participants' individual performances were compared over the three learning conditions regarding:

- Task completion time.

- Planning landmarks: the use of fewer landmarks indicates that radiologists rely more on haptic feedback than on visual feedback to reach the target (he uses haptic perception to detect a change in layer compliance). Hence, the participants were asked to minimize the number of landmarks.

- Organ penetrations and contact with organs: the radiologist tries to avoid lesions and to minimize damage to the organs. Hence, the participants were asked to minimize organs damage by minimizing the number of organ penetrations and contact with the organs.

- Length of the real insertion path: in order to limit damage to the body, participants were asked to minimize the insertion path length. When inserting the needle, participants did not necessarily respect the planned path. Hence, we calculated the length of the real insertion path length rather than the planned path length.

- Frequency of missed targets: The number of times the participant missed the target organ.

- Final distance to the target: the radiologists aim to position the needle tip in the center of the target.

- Insertion segments: When performing a biopsy, radiologists split their gestures into several small insertion movements (segments). The number of segments and the 
average amplitude of the segments were used to characterize the learners' needle insertion profiles.

Furthermore, the insertion strategy for one practice exercise was investigated. This was used to determine the components that influenced task performance according to the learning method.

Finally, each participant answered a questionnaire after the practice session. The questionnaire was used as a subjective measure for the system evaluation.

\section{RESULTS AND DISCUSSION}

The statistical significance of the data was evaluated using ANOVA ( $F$ values are provided) and pair-wise $t$-tests ( $t_{\text {DoF }}$ values are provided). Frequencies were compared using the Chisquare test $\left(\chi^{2}\right.$ values are provided). The results are summarized and discussed hereafter (significant values are presented in bold within the text and the data tables):

\subsection{Performance}

\subsubsection{Task completion time}

The results (Table 2) show that the participants performed the task faster in the VHI condition compared to participants in the PI condition $\left(\mathbf{t}_{\mathbf{3 8}}=\mathbf{2 . 8} ; \mathbf{p}<\mathbf{0 . 0 5}\right)$ and in the VI condition $\left(\mathbf{t}_{\mathbf{3 8}}=\mathbf{4 . 8} ; \mathbf{p}<\mathbf{0 . 0 5}\right)$. No significant difference was observed between participants in the PI and VI groups $\left(\mathrm{t}_{38}=1.7 ; \mathrm{p}>0.05\right)$. In order to determine more accurately the causes of this decrease in completion time, additional analyses were conducted (Table 2):

- Planning time: No significant differences were observed among the three groups.

- Manipulation time: the needle insertion time of the participants in the VHI condition was less than those in the PI condition $\left(\mathbf{t}_{\mathbf{3 8}}=\mathbf{3 . 1} ; \mathbf{p}<\mathbf{0 . 0 5}\right)$ and in the VI condition $\left(\mathbf{t}_{\mathbf{3 8}}=\right.$ 3.5; $\mathbf{p}<\mathbf{0 . 0 5}$ ). No significant difference was observed between the PI and VI groups $\left(\mathrm{t}_{38}=0.9 ; \mathrm{p}>0.05\right)$.

Table 2: task completion time

\begin{tabular}{|c|c|c|c||c||}
\hline$*=\mathbf{p}<\mathbf{0 . 0 5}$ & $\begin{array}{c}\text { PI : mean } \\
(\text { SD })\end{array}$ & $\begin{array}{c}\text { VI: mean } \\
(\text { SD })\end{array}$ & $\begin{array}{c}\text { VHI: mean } \\
(\text { SD })\end{array}$ & $\begin{array}{c}\text { F-values } \\
(\mathbf{2 , 5 7})\end{array}$ \\
\hline Total time (seconds) & $2243.2(639.5)$ & $2628.5(579.4)$ & $\mathbf{1 8 7 6 . 1}(\mathbf{3 4 3 . 7 )}$ & $\mathbf{7 . 9 3 8 *}$ \\
\hline Planning time (seconds) & $904.8(323.1)$ & $1037.3(789.5)$ & $789.5(252.3)$ & 2.590 \\
\hline Manipulation time (seconds) & $673.5(201.5)$ & $743.9(271.8)$ & $\mathbf{4 9 1 . 3}(\mathbf{1 5 6 . 8})$ & $\mathbf{6 . 9 6 3 *}$ \\
\hline
\end{tabular}

This result supports our hypothesis claiming that the visual-haptic learning promotes faster motor skills performance. However, this is not only due to the speed of movement differences between the participants; other elements also influenced the time difference as discussed in the following section.

\subsubsection{Accuracy}

The accuracy is evaluated using different measures:

Table 3: comparison of participants' performances

\begin{tabular}{|l|c|c|c||c|}
\hline$*=\mathbf{p}<0.05$ & $\begin{array}{c}\text { PI : mean } \\
(\text { SD })\end{array}$ & $\begin{array}{c}\text { VI: mean } \\
(\text { SD })\end{array}$ & $\begin{array}{c}\text { VHI: mean } \\
(\text { SD })\end{array}$ & F-values $(2,57)$ \\
\hline
\end{tabular}




\begin{tabular}{|c|c|c|c|c|}
\hline $\begin{array}{l}\text { Planning landmarks } \\
\text { (landmarks) }\end{array}$ & $12.1(6.3)$ & $15.3(10.4)$ & $8.5(4.3)$ & 4.054* \\
\hline Organ contacts (contacts) & $32.9(29.0)$ & $31.9(28.8)$ & $15.7(15.2)$ & $2.799 *$ \\
\hline Organ penetrations & $3.3(0.9)$ & $3.5(0.8)$ & $2.1(0.7)$ & $16.7^{*}$ \\
\hline Length of the real path $(\mathrm{cm})$ & $54.6(20.3)$ & $64.2(23.7)$ & $39.7(22.7)$ & $5.89 *$ \\
\hline Target missing (\%) & $80 \%$ & $60 \%$ & $30 \%$ & $\begin{array}{c}\chi_{\text {obs }}^{2}=\mathbf{6 . 1 7}> \\
\chi_{(0.05 ; 2)}^{2}=10.3\end{array}$ \\
\hline Distance to the target $(\mathrm{cm})$ & $0.68(0.18)$ & $0.71(0.18)$ & $0.63(0.13)$ & 0.98 \\
\hline
\end{tabular}

\section{Number of planning landmarks}

The results (Table 3) show that the participants used fewer landmarks to plan the insertion path in the VHI condition as compared to participants in PI condition $\left(\mathbf{t}_{\mathbf{3 8}}=\mathbf{2 . 1} ; \mathbf{p}<\mathbf{0 . 0 5}\right)$ and VI condition $\left(\mathbf{t}_{\mathbf{3 8}}=\mathbf{2 . 6} ; \mathbf{p}<\mathbf{0 . 0 5}\right)$. No significant difference was observed between participants in the PI and VI groups $\left(\mathrm{t}_{38}=1.1 ; \mathrm{p}>0.05\right)$.

\section{Contact with the organs}

The results (Table 3) show that the participants in the VHI condition has the least contact with the organs as compared to participants in the PI condition $\left(\mathbf{t}_{\mathbf{3 8}}=\mathbf{2 . 2} ; \mathbf{p}<\mathbf{0 . 0 5}\right)$ and in the VI condition $\left(\mathbf{t}_{\mathbf{3 8}}=\mathbf{2 . 1} ; \mathbf{p}<\mathbf{0 . 0 5}\right)$. No significant difference was observed between participants in the PI and VI groups $\left(\mathrm{t}_{38}=0.1 ; \mathrm{p}>0.05\right)$.

\section{Needle penetrations inside the organs}

The results (Table 3) show that the participants penetrated the organs less often in the VHI condition compared to participants in the PI condition $\left(\mathbf{t}_{\mathbf{3 8}}=\mathbf{4 . 5} ; \mathbf{p}<\mathbf{0 . 0 5}\right)$ and in the VI condition $\left(\mathbf{t}_{\mathbf{3 8}}=\mathbf{5 . 8} ; \mathbf{p}<\mathbf{0 . 0 5}\right)$. No significant difference was observed between participants in the PI and VI groups $\left(\mathrm{t}_{38}=-0.5 ; \mathrm{p}>0.05\right)$.

\section{The length of the real insertion path}

The results (Table 3) show that the participants reduces the length of the insertion path in the VHI condition compared to participants in the PI condition $\left(\mathbf{t}_{\mathbf{3 8}}=\mathbf{2 . 1} ; \mathbf{p}<\mathbf{0 . 0 5}\right)$ and the VI condition $\left(\mathbf{t}_{\mathbf{3 8}}=\mathbf{3 . 2} ; \mathbf{p}<\mathbf{0 . 0 5}\right)$. No significant difference was observed between participants in the PI and VI groups $\left(\mathrm{t}_{38}=-1.3 ; \mathrm{p}>0.05\right)$.

\section{Frequency of missed targets}

All subjects correctly reached the targets; however, some of them needed more than one trial to succeed. Results show that the participants missed the targets less frequently in the VHI condition compared to participants in the PI and VI conditions.

\section{Final distance to the target}

As shown above, all the participants correctly completed the task by reaching the target. The results (Table 3) show no significant difference between participants of the three learning groups concerning the final distance between the needle tip and the center of the target.

Even if the results show no evident difference between groups concerning the final distance to the target, the other measures indicate that the participants were more accurate after the visual-haptic learning. On the other hand, the participants were less accurate after the paper instruction learning and the visual learning. 
This can explain the decrease of the completion time for the VHI group. The participants avoided the insertion constraints, helping them to reach the target more quickly than participants in the two other groups: they master the characteristics of the skills better through this learning method compared to the conventional biopsy leaning methods. This confirms the hypothesis $\mathbf{H 1}$.

\subsection{Subjective evaluation}

Participants answered a (yes/no set of questions) questionnaire to evaluate their perception of the use of the learning system. The Chi-square test (Table 4) shows no significant differences between groups regarding this questionnaire answers.

We were expecting the visual-haptic learning to help the users perceive the system as easier to use. However, the high global evaluation scores indicate that all of the participants (independently from the learning method) appreciated the use of the learning system.

One possible explanation is that the use of VR technology and haptic devices provided a newer and richer interactive environment for the students when compared to conventional learning environments. This influences the users' perception of the learning environment, as none of them were familiar with the use of these technologies. Further investigations must be completed to better understand the user's perception of the system. Hence, the hypothesis $\mathbf{H} 2$ is rejected.

Table 4: questionnaire answers

\begin{tabular}{|c|c|c|c||c||}
\hline Perception of the ease of: & PI (\%) & VI (\%) & VHI (\%) & $\begin{array}{c}\chi^{2} \text { values compared to } \\
\chi_{(0.05 ; 2)}^{2}=5.99\end{array}$ \\
\hline Performing the exercises & $75 \%$ & $86.7 \%$ & $70 \%$ & $\chi_{\text {obs }}^{2}=1.35<5.99$ \\
\hline Manipulating the haptic device & $90 \%$ & $73.3 \%$ & $65 \%$ & $\chi_{\text {obs }}^{2}=3.57<5.99$ \\
\hline Orienting the needle & $70 \%$ & $66.7 \%$ & $60 \%$ & $\chi_{\text {obs }}^{2}=0.46<5.99$ \\
\hline Applying pressure on the needle & $95 \%$ & $86.7 \%$ & $80 \%$ & $\chi_{\text {obs }}^{2}=2.03<5.99$ \\
\hline Understanding the user interface & $95 \%$ & $93 \%$ & $95 \%$ & $\chi_{\text {obs }}^{2}=0.1<5.99$ \\
\hline Working in the environment & $80 \%$ & $80 \%$ & $65 \%$ & $\chi_{\text {obs }}^{2}=1.5<5.99$ \\
\hline
\end{tabular}

\subsection{Needle insertion profiles}

\subsubsection{The number of insertion segments}

The results (Table 5) show that the participants increased the number of insertion segments in the VI condition compared to the PI condition $\left(\mathbf{t}_{\mathbf{3 8}}=\mathbf{2 . 8} ; \mathbf{p}<\mathbf{0 . 0 5}\right)$ and the VHI conditions $\left(\mathbf{t}_{\mathbf{3 8}}=\mathbf{4 . 2} ; \mathbf{p}<\mathbf{0 . 0 5}\right)$. No significant difference was observed between participants in the PI and VHI groups $\left(\mathrm{t}_{38}=0.9 ; \mathrm{p}>0.05\right)$.

\subsubsection{Average amplitudes of the needle insertion segments}

The results (Table 5) show that the participants increased the length of the insertion segments in the PI condition compared to participants in the VI condition $\left(\mathbf{t}_{\mathbf{3 8}}=\mathbf{2 . 2} ; \mathbf{p}<\mathbf{0 . 0 5}\right)$ and VHI conditions $\left(\mathbf{t}_{\mathbf{3 8}}=\mathbf{2 . 1} ; \mathbf{p}<\mathbf{0 . 0 5}\right)$. No significant difference was observed between the VI and VHI groups $\left(\mathrm{t}_{38}=0.2 ; \mathrm{p}>0.05\right)$.

Table 5: comparison of needle insertion profiles 


\begin{tabular}{|c|c|c|c||c|}
\hline$*=\mathbf{p}<\mathbf{0 . 0 5}$ & $\begin{array}{c}\text { PI : mean } \\
(\mathbf{S D})\end{array}$ & $\begin{array}{c}\text { VI: mean } \\
(\mathbf{S D})\end{array}$ & $\begin{array}{c}\text { VHI: mean } \\
(\mathbf{S D})\end{array}$ & F-values $(\mathbf{2 , 5 7 )}$ \\
\hline $\begin{array}{c}\text { Insertion segments number } \\
\text { (segments) }\end{array}$ & $69.4(28.2)$ & $\mathbf{9 6 . 1}(\mathbf{3 0 . 0})$ & $62.2(18.4)$ & $\mathbf{8 . 9}^{*}$ \\
\hline Insertion segments amplitudes $(\boldsymbol{C m})$ & $\mathbf{2 . 5}(\mathbf{1 . 0})$ & $2.1(1.0)$ & $2.1(0.8)$ & $\mathbf{3 . 6}^{*}$ \\
\hline
\end{tabular}

Accordingly, three needle manipulation profiles were extracted:

- PI group profile: The novices in this group did not have a visual demonstration of the movement dynamics, and therefore did not learn the correct movement amplitudes. Consequently, they moved the needle using longer segments.

- VI group profile: compared to the PI group participants, the VI group participants decreased the size of insertion segments. Indeed, they had a real-time visual feedback of the movement. Visual demonstrations helped them to acquire the correct size of the movement segments.

On the other hand, the PI and the VI groups missed the target more frequently. Subsequently, they increased the size of the insertion path. To correctly reach the target they used two different strategies; the PI group participants divided their movements into bigger and fewer segments while the VI group participants used more segments with smaller amplitudes.

- VHI group profile: The real time visual feedback of the correct movement allows the learners to correctly perform the movement. Since the participants in this group reached the target with fewer attempts, they also reduced the number of insertion segments.

\subsection{The insertion strategy}

A more in-depth analysis of the insertion profiles was conducted for one scenario. The scenario was chosen according to the cognitive task analysis because it allows for observation of different insertion strategies as shown in the following description (Erreur ! Source du renvoi introuvable.):

The target was placed under a "green" organ (participants were allowed to penetrate green organs). The easiest strategy would be to insert the needle vertically to reach the target as quick as possible while avoiding penetration angles. However, a vein was placed above the organ and the target. Furthermore, a "red" organ was placed to the right of the green organ. Since penetrating veins and red organs was forbidden, the learners had to consider these constraints when choosing the optimal strategy.

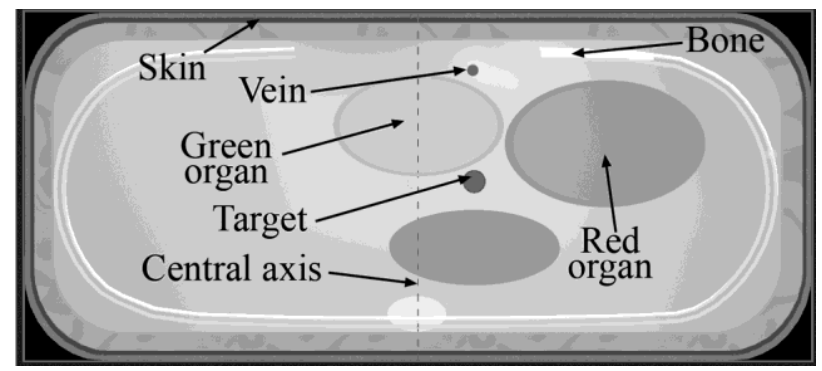


Fig. 9: The scenario description: two possible paths; the needle could be inserted between the bone and the vein to reach the target from the right avoiding the red organ; or it can be inserted on the left of the vein to penetrate the green organ and then reach the target

Two different insertion strategies were observed (depending on the needle entry point):

The "Left-strategy": participants chose this strategy to avoid the vein by passing on the left side and tilting the needle to the left. The advantage of choosing this strategy is that the needle can be inserted far enough away from the red organ. It also allows for more space in order to avoid the vein (there were no bones to block the needle).

The Right-strategy: participants chose this strategy to avoid the vein by passing on the right side and tilting the needle to the right. The advantage of choosing this strategy is that it allows the needle to be inserted with an angle to the right. This seems to be more intuitive and easier to perform than an angle to the left (for right-handed persons). Indeed, the right orientation is more comfortable according to the posture of the hand holding the needle (e.g., the position of the pen when writing). However, there is less space in which to avoid the vein in this case; the bone constrains the penetration angle. Furthermore, there is a risk of penetrating the red organ. This requires more accuracy to correctly insert the needle.

\subsubsection{Strategy-based performance}

The results show that $44.83 \%$ of the participants missed the target when they chose the leftstrategy. On the other hand, only $\mathbf{1 6 . 1 3 \%}$ of them missed the target when they chose the right-strategy $\left(\chi_{\text {obs }}^{2}=\mathbf{5 . 7 8}>\chi_{\mathbf{t}}^{2}(\mathbf{0 . 0 5} ; \mathbf{1})=\mathbf{3 . 8 4}\right)$. No significant differences were found regarding the frequency of forbidden organs penetration $(17.86 \%$, left-strategy; $12.50 \%$, right-strategy; $\left.\chi^{2} \mathrm{Obs}=1.56<\chi_{\mathrm{t}(0.05 ; 1)}^{2}=3.84\right)$.

\subsubsection{Learning groups performance}

\section{The choice of the insertion strategies}

Results (Table 6) show that the choice of the strategy is significantly dependent on the learning method $\left(\chi_{\text {Obs }}^{\mathbf{2}}=\mathbf{8 . 4 0}>\chi_{\mathrm{t}}^{2}(0.05 ; 2)=5.99\right)$. The majority of PI group participants chose the left-strategy compared to the two other groups. On the other hand, the majority of VHI group participants chose the right-strategy compared to the two other groups.

Table 6: Performance OF the investigated scenario

\begin{tabular}{|c|c|c|c|c|}
\hline$*=p<0.05$ & $\begin{array}{l}\text { PI : mean } \\
\quad(\mathrm{SD})\end{array}$ & $\begin{array}{l}\text { VI: mean } \\
\quad(\text { SD) }\end{array}$ & $\begin{array}{c}\text { VHI: mean } \\
\text { (SD) }\end{array}$ & F-values $(2,57)$ \\
\hline Organ penetration $(\mathrm{cm})$ & $8.3(1.7)$ & $8.4(1.8)$ & $7.1(2.0)$ & 3.7 \\
\hline $\begin{array}{c}\text { Contacts with the green } \\
\text { organ }\end{array}$ & $25.2(14.5)$ & $23.7(16.8)$ & $13.9(8.6)$ & 3.7 \\
\hline Target missing (\%) & $50 \%$ & $45 \%$ & $15 \%$ & $\chi_{\text {obs }}^{2}=6.17>\chi_{(0.05 ; 2)}^{2}=5.99$ \\
\hline $\begin{array}{c}\text { Forbidden organs } \\
\text { penetration }(\%)\end{array}$ & $20 \%$ & $15 \%$ & $10 \%$ & $\chi_{\text {obs }}^{2}=0.78<\chi_{(0.05 ; 2)}^{2}=5.99$ \\
\hline
\end{tabular}

\section{Number of organ penetrations}

Results (Table 6) show that participants in the VHI condition minimized the amount of organ penetrations as compared to the PI condition $\left(\mathbf{t}_{\mathbf{3 8}}=\mathbf{2 . 2} ; \mathbf{p}<\mathbf{0 . 0 5}\right)$ and the VI condition $\left(\mathbf{t}_{38}=\right.$ 
2.4; $\mathbf{p}<\mathbf{0 . 0 5})$. No significant difference was observed between participants in the PI and VI groups $\left(\mathrm{t}_{38}=0.5 ; \mathrm{p}>0.05\right)$.

\section{Contact with the green organ}

Results (Table 6) show that the participants decreased the number of contacts with organs in the VHI condition compared to participants in the PI condition $\left(\mathbf{t}_{\mathbf{3 8}}=\mathbf{2 . 9} ; \mathbf{p}<\mathbf{0 . 0 5}\right)$ and in the VI condition $\left(\mathbf{t}_{\mathbf{3 8}}=\mathbf{2 . 2} ; \mathbf{p}<\mathbf{0 . 0 5}\right)$. No significant difference was observed between the PI and VI groups $\left(\mathrm{t}_{38}=0.2 ; \mathrm{p}>0.05\right)$.

\section{Target missing}

Results (Table 6) show that the participants in the VHI group missed the target less frequently than participants in the PI and in the VI groups.

\section{Forbidden organs penetration}

No differences were found among groups for the frequency of the forbidden organs penetration (Table 6).

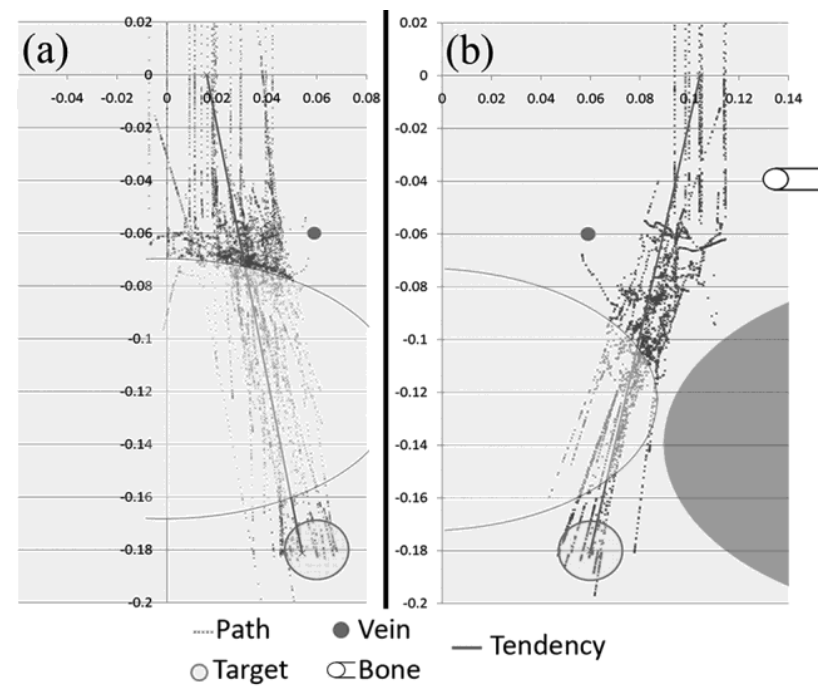

Fig. 10: Proportions of insertion strategies for the PI group: (a) left strategy (70\%), (b) right strategy (30\%). 50\% missed the target

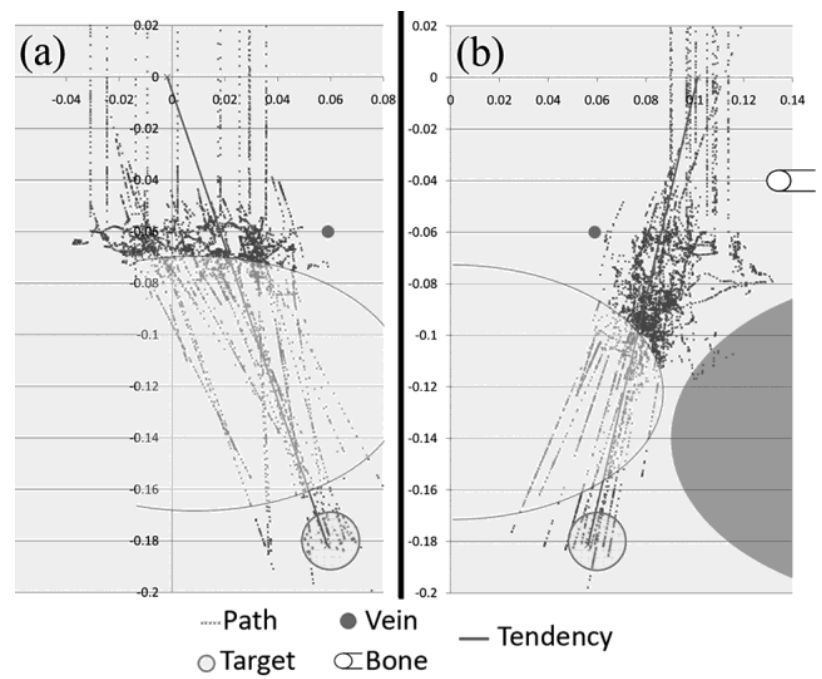

Fig. 11: Proportions of insertion strategies for the VI group: (a) left strategy (55\%), (b) right strategy (45\%). 45\% missed the target 


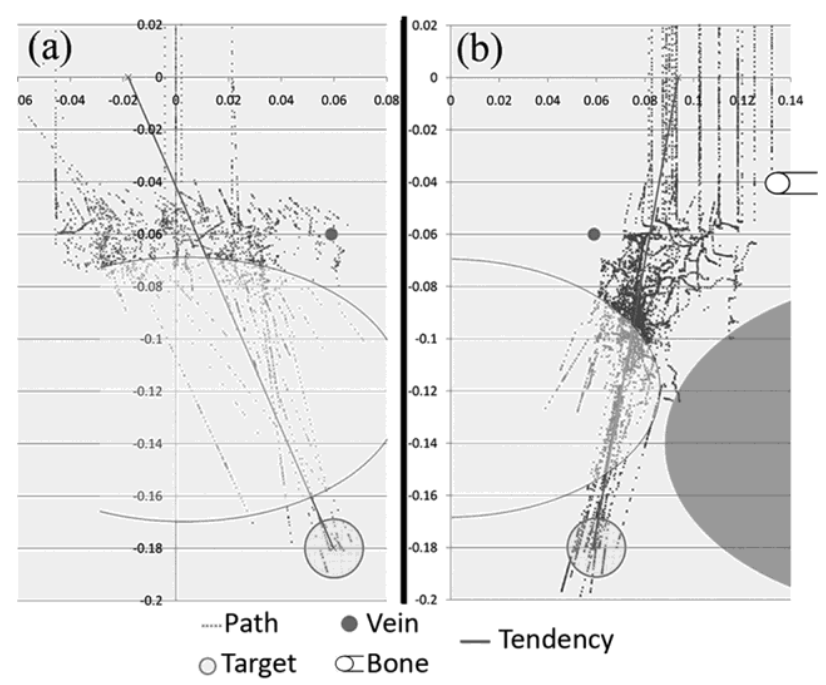

Fig. 12: Proportions of insertion strategies for the VHI group: (a) left strategy (25\%), (b) right strategy (75\%). 15\% missed the target

Erreur ! Source du renvoi introuvable., Erreur ! Source du renvoi introuvable., and Erreur! Source du renvoi introuvable. show the real insertion paths for participants according to the learning group and the chosen strategy. These figures show the positions of the different organs (the red organ, the green organ, the bone, the vein, and the tumor). The " 0 " on the $\mathrm{x}$-axis represents the central axis of the body while the " 0 " on the y-axis represents the skin surface. These figures give a more clear idea of the participants' performance according to the chosen strategies (amount of organ penetration, target missing, and forbidden organs penetration). This will be used to determine and discuss the optimal strategy for this scenario.

\subsubsection{The optimal strategy}

The results indicate that the right-strategy:

- Was no safer than the left-strategy.

- Allows the users to reach the target more accurately

- Allows the needle to be inserted with an angle to the right that was more intuitive and easier to perform.

All these elements suggest that the right-strategy is the optimal insertion strategy for this scenario.

The majority of VHI group participants chose the optimal strategy. This suggests that the visual-haptic learning helps them to better perceive the complexity of the needle manipulation. Hence, they were aware that inclining the needle to the right allows for easier manipulation. Furthermore, they were more confident when performing the task. Indeed, they chose the strategy that requires more accuracy to perform the needle insertion. However, this does not prevent them from having the best performances in this scenario. This suggests that the visual-haptic method allows them to focus on the learning of the correct properties of the motor skill.

On the other hand, the majority of PI preferred to be careful by choosing the strategy that minimizes the risks of penetrating the forbidden organs (the left strategy). This suggests that 


\section{the paper instruction learning encourages them to focus on the theoretical rules}

(declarative knowledge) rather than on the motor skill properties. However, the complexity of the needle manipulation with the left angle penetration prevents them from having a good performance. This suggests that the theoretical learning method does not provide novices with the basic components needed for optimal needle insertion, but allowed them to better integrate the theoretical rules.

Finally, the VI group participants chose equally between both strategies. This suggests that visual learning does not provide enough knowledge required to choose the optimal strategy. However, the visual feedback allows for transmission of the correct dynamics of the movement.

These findings confirm our hypothesis H3.

\section{CONCLUSION}

In this paper, we investigated a teaching method for biopsy needle insertion based on haptic communication in virtual environments. A communication paradigm based on collaborative training was used to support haptic interactions between a skilled-operator and a novice. A virtual environment for performing the biopsy task has been developed to examine the effects of such a paradigm on learning in the early-stage of knowledge transfer. The paradigm was compared to existing teaching methods for biopsy procedures.

\subsection{Findings}

The results indicate that the different learning methods have permitted to teach different task characteristics. While all the novices have received the same information during learning (insertion rules and manipulation constraints), they have put forward different aspects of the task during the practice session. Therefore, the cognitive skills (the rules) were used differently depending on the knowledge acquired by each group. However, the haptic-visual learning has an added value compared to the traditional leaning methods for biopsy. Indeed, it helps the learners to focus on technical skills: the constraints of positioning and the manipulability of the tool which are very relevant to this task. This gives the learners a more complete representation of the situation allowing them to adapt their strategy to the actual context. Subsequently, they had the best performances for the virtual biopsy task. On the other hand, the other novices were focusing on other aspects of the task that depended on their learning method. This shows that if one does not use the appropriate communication channel, the information is not well integrated by the learners.

We conclude that the choice of the appropriate sensory channel to be used to communicate the information to the learners must depend on the task and on the nature of the knowledge we want to teach in order to give them a more complete representation of the situation. For that purpose, our task-centered design methodology with the complete task analysis was efficient to reveal in advance the relevant information for the biopsy procedure and the appropriate channel that must be used to transmit this information.

\subsection{Design implications}

The user study shows that the system improves the performance of the users. Some recommendations can be given for motor skills learning systems design: 
1. Depending on the task, the design of the system must consider the importance of haptic interactions.

2. In order to be efficient, a motor skills learning system must support haptic communication. This communication channel helps an expert transmit inexpressible knowledge to the learners.

3. In order to be efficient, the haptic channel must be combined with other communication channels.

These recommendations can permit to design systems that match better the users' needs in terms of knowledge transfer.

\subsection{Limitations and Future Work}

We are continuing the investigation of the haptic communication paradigm in collaborative virtual environments. We think that the paradigm can be used in other learning scenarios. One can imagine a learning scenario in which an expert practitioner can supervise trainees during practice. This scenario can allow the novices to become more active and to learn from their own mistakes. Furthermore, since the teacher follows the novices' actions, he can give practical recommendations to the students, allowing for more efficient training of novice practitioners. This learning scenario can then be combined with the actual scenario in order to improve the learning.

On the other hand, our system can transmit information about positions but not about the forces. Indeed, the real time implementation of the communication paradigm does not allow the expert to share information about the forces that were applied. Methods (Gillespie, O'Modhrain, Tang, Zaretsky, \& Pham, 1998; Dang, Annaswamy, \& Srinivasan, 2001) that allow for transmission of both position and force data simultaneously are not applicable. One possible solution would be to have two learning sessions in which position and force are taught separately, or to combine both force and position feedback at a high rate so that two collaborating users can feel the same, as stated in (Chellali, Dumas, \& Milleville-Pennel, 2011). In order to evaluate these approaches, two new studies for spatial guidance (Takac, Chellali, Dumas, Milleville-Pennel, Grosdemouge, \& Cao, 2011) and the training for the perception of forces (Roth Monzon, Chellali, Dumas, \& Cao, 2012) are being conducted.

The actual study demonstrates the efficacy of the new collaborative haptic training method for biopsy procedures. However, further investigations are needed to assess its applicability to learn other surgical skills.

Finally, our study did not investigate the effects of haptic communication neither on skills retention, nor on the transfer of the acquired knowledge to the real world situation. These additional steps are necessary in order to use such a system to train future radiologists.

\section{ACKNOWLEDGMENT}

This work was conducted in collaboration with the Teaching Hospital of Nantes and is supported in part by a grant from Orange Labs. The authors would like to thank all of the volunteers who participated in the experiment. 


\section{REFERENCES}

Baldwin, P., Paisley, A., \& Brown, S. (1999). Consultant surgeons' opinion of the skills required of basic surgical trainees. British Journal of Surgery, 86, 1078-1082.

Barbé, L., Bayle, B., \& de Matheli, M. (2006). Bilateral controllers for teleoperated percutaneous interventions : evaluation and improvements. In Proceedings of the American Control Conference ACC'06.

Basdogan, C., Ho, C., Srinivasan, M. A., \& Slater, M. (2000). An experimental study on the role of touch in shared virtual environments. ACM Transactions on Computer-Human Interaction, 7 (4), 443-460.

Blavier, A. (2006). Impact des images en 2D ou 3D sur les processus cognitifs impliqués dans le traitement visuel et dans le contrôle de l'action : le cas de la chirurgie minimale invasive (Doctoral dissertation). Retrieved from http://hdl.handle.net/2268/28282.

Bluteau, J., Coquillart, S., Payan, Y., \& Gentaz, E. (2008). Haptic guidance improves the visuo-manual tracking of trajectories. PLoS ONE, 3 (3), e1775.

Burdea, G., \& Coiffet, P. (2003). Virtual reality technology. New York: Wiley-IEEE Press.

Cao, C. (2001). Designing for spatial orientation in non-rigid endoscopic environments. In Proceedings of the 45th Annual Meeting of the Human Factors and Ergonomics Society, (pp. 1259-1263).

Chellali, A., Dumas, C., \& Milleville-Pennel, I. (2011). Influences of Haptic Communication on a Shared Manual Task in a Collaborative Virtual Environment. Elsevier Interacting With Computers, 23 (4), 317-328.

Chellali, A., Milleville, I., \& Dumas, C. (2010). WYFIWIF: A haptic communication paradigm FOR collaborative motor skills learning. In Proceedings of the International Conference on Web Virtual Reality and $3 D$ worlds 2010 (pp. 301-308).

Clark, H., \& Brennan, S. (1991). Grounding in Communication. In L. Resnick, J. Levine, \& S. Teasley (Eds.), Cognition, Perspectives on Socially Shared (pp. 127-149). Washington D.C.: American Psychological Association.

Cuschier, A. (2011). What do master surgeons think of surgical competence and revalidation? American Journal of Surgery, 182, 110-116.

Dang, T., Annaswamy, T. M., \& Srinivasan, M. A. (2001). Development and Evaluation of an Epidural Injection Simulator with Force Feedback for Medical Training. In Proceedings of Medicine Meets Virtual Reality, (pp. 97-102).

Esen, H., Sachsenhauser, A., Yano, K., \& Buss, M. (2007). Multi-User Virtual Training System Concept and Objective Assessment of Trainings. In Proceedings of the 16th IEEE International Symposium on Robot and Human Interactive Communication, (pp. 1084-1089).

Feygin, D., Keehner, M., \& Tendick, F. (2002). Haptic Guidance: Experimental Evaluation of a Haptic Training Method for a Perceptual Motor Skill. In Proceedings of the 10th Symposium on Haptic Interfaces for Virtual Environment and Teleoperator Systems: HAPTICS'02, (pp. 40-47).

Gallagher, A., McClure, N., McGruigan, J., Crothers, I., \& Browning, L. (1999). Virtual reality training in laparoscopic surgery: a preliminary assessment of Minimally Invasive Surgical Trainer Virtual Reality (MIST VR). Endoscopy 1999, 31 (4), 310-313.

Gillespie, R. B., O'Modhrain, M., Tang, P., Zaretsky, D., \& Pham, C. (1998). The virtual teacher. In Proceedings of the ASME International Mechanical Engineering Conference and Exposition.

Gunn, C., Hutchins, M., \& Adcock, M. (2005). Combating latency in haptic collaborative virtual environments. Presence: Teleoperators and Virtual Environments, 14 (3), 313-328.

Hutchins, M., Adcock, A., Stevenson, D., Gunn, C., \& Krumpholz, A. (2005). The design of perceptual representations for practical networked multimodal virtual training environments. In Proceedings of the 11th International Conference on Human-Computer Interaction: HCI International '05.

Lathan, C., Cleary, K., \& Traynor, L. (2000). Human-Centered Design of a Spine Biopsy Simulator and the Effects of Visual and Force Feedback on Path-Tracking Performance. Presence: Teleoperators and Virtual Environments, 9 (4), 337-349. 
Lee, J., \& Choi, S. (2010). Effects of Haptic Guidance and Disturbance on Motor Learning: Potential Advantage of Haptic Disturbance. In Proceedings of the Haptics Symposium 2010, (pp. 335-342).

Lim, S. (2002). The Self-Confrontation Interview: Towards an Enhanced Understanding of Human Factors in Web-based Interaction for Improved Website Usability. The Journal of Electronic Commerce Research, 3 (3), $162-170$.

Liukkonen, T. N. (2010). Human Reaction Times as a Response to Delays in Control Systems. Retrieved Jan. 24, 2012, from http://www.measurepolis.fi:

http://www.measurepolis.fi/alma/ALMA\%20Human\%20Reaction\%20Times\%20as\%20a\%20Response $\% 20$ to $\%$ 20Delays\%20in\%20Control\%20Systems.pdf

Merians, A., Poizner, H., Boian, R., Burdea, G., \& Adamovich, S. (2006). Sensorimotor training in a virtual reality environment: does it improve functional recovery poststroke? Neurorehabil Neural Repair, 20 (2), 252267.

Morris, D., Tan, H., Barbagli, F., Chang, T., \& Salisbury, K. (2007). Haptic Feedback Enhances Force Skill Learning. In proceedings of EuroHaptics: Conference and Symposium on Haptic Interfaces for Virtual Environment and Teleoperator Systems, (pp. 21-26).

Nudehi, S., Mukherjee, R., \& Ghodoussi, M. (2003). A Haptic Interface Design for Minimally Invasive Telesurgical Training and Collaboration in the presence of Time Delay. In Proceedings of the 42nd IEEE conference on decision and control.

Payne, S. J. (2009). Mental models in Human Computer Interaction. In A. Sears \& J. A. Jacko (Ed.), Humancomputer interaction: Fundamentals (pp. 39-52). Boca Raton: CRC Press.

Rasmussen, J. (1983). Skills, rules, knowledge: signals, signs and symbols and other distinctions in human performance models. IEEE transactions on Systems, Man, and

Cybernetics, 13 (3), 257-266.

Roth Monzon, E. L., Chellali, A., Dumas, C., \& Cao, C. (2012). Training effects of a visual aid on haptic sensitivity in a needle insertion task. In the proceedings of the IEEE Haptics symposium, (pp. 199-202).

Sallnäs, E.-L., Rassmus-Gröhn, K., \& Sjöström, C. (2000). Supporting presence in collaborative environments by haptic force feedback. ACM Transactions on Computer-Human Interaction, 7 (4), 461-476.

Solis, J., Avizzano, C., \& Bergamasco, M. (2003). Validating a skill transfer system based on reactive robots technology. In Proceedings of The 12th IEEE International Workshop onRobot and Human Interactive Communication, ROMAN'03, (pp. 175-180).

Stassen, H. G., Bonjer, H. J., Grimbergen, C. A., \& Dankelman, J. (2005). The Future of Minimally Invasive Surgery and Training. In J. Dankelman, C. A. Grimbergen, \& H. G. Stassen (Eds), Engineering for patient safety: issues in minimally invasive procedures (pp. 272-282). London: Lawrence Erlbaum Associates Inc.

Takac, B. (2010). Haptic communication in virtual environments (European Master in Advanced Robotics Thesis). Ecole Centrale de Nantes, Nantes, France.

Takac, B., Chellali, A., Dumas, C., Milleville-Pennel, I., Grosdemouge, C., \& Cao, C. (2011). Haptic Communication for a 2-D Pointing Task in a Virtual Environment. In proceedings of the 55th Annual Meeting of the Human Factors and Ergonomics Society, (pp. 2168-2172).

Willingham, D. B. (1998). A neuropsychological theory of motor skill learning. Psychological Review, 105 (3), 558-584.

Yoshikawa, T., \& Henmi, K. (2000). Human Skill Transfer Using Haptic Virtual Reality Technology. In Proceedings of the 6th international Symposium on Experimental Robotics VI, 250, (pp. 351-360).

Zhou, M., Tse, S., Derevianko, A., Jones, D., Schwaitzberg, S., \& Cao, C. (2008). The Effect of Haptic Feedback on Laparoscopic Suturing and Knot-tying: A Learning Curve Study. In Proceedings of the 52nd Annual Meeting of the Human Factors and Ergonomics Society, (pp. 880-884). 